\title{
DETERMINAÇÃO DOS HIDROCARBONETOS SATURADOS E POLICÍClICOS AROMÁTICOS PRESENTES NO MATERIAL PARTICULADO DA ATMOSFERA AMAZÔNICA
}

Pérola de Castro Vasconcellos

Instituto de Química - Universidade de São Paulo

Paulo Eduardo Artaxo

Instituto de Física - Universidade de São Paulo

Paulo Ciccioli, Angelo Cecinato, Enzo Brancaleoni e Massimiliano Frattoni

Instituto de Poluição Atmosférica - Conselho Nacional de Pesquisa - Roma - Itália

Recebido em 19/12/96; aceito em 22/12/97

\begin{abstract}
CHEMICAL COMPOSITION OF AEROSOL COLLECTED IN THE AMAZON FOREST. It was identified and quantified several organic compounds in the atmosphere of a site into Amazon Basin with high impact of biomass burning emission. It was important to know the particulate matter composition with respect to n-alkanes and PAH associated with the particulate matter because they provided indication on the main sources contributing to airborne particles, the contribution of natural vs. man-made emission and the aging of the particles. The main classes of compounds observed were n-alkanes, PAH and nitro-PAH. It was observed the formation of nitroPAH from photochemical reactions. The aerosol mass concentration is mainly associated with fluoranthene, pyrene and benzo(ghi)perylene. Environmental and direct emissions samples (flaming and smoldering) were collected and analysed.
\end{abstract}

Keywords: biomass burning; atmospheric organic mater; polycyclic compounds.

\section{INTRODUÇÃO}

O estudo de aerossóis coletados em áreas remotas tem recebido considerável atenção nas últimas décadas, entretanto, o conhecimento da composição química do aerossol atmosférico em escala global ainda é limitado.

Uma região importante para estudar os processos que estão mudando a composição da atmosfera global é a floresta tropical Amazônica devido a grande quantidade de aerossóis emitida durante a queima de biomassa.

As emissões de fumaça e partículas afetam a formação das nuvens e as propriedades ópticas da atmosfera. Os gases traços emitidos pela queima reagem nas fases aquosas e gasosas, influenciando a capacidade oxidativa da atmosfera e o nível de $\mathrm{O}_{3}$ em particular ${ }^{1}$.

Outras substâncias emitidas em grande quantidade pela queima de biomassa, pertencem à classe química dos hidrocarbonetos policíclicos aromáticos (HPAs). Elas exibem um efeito indutor cancerígeno em várias espécies de animais e são suspeitas de causar câncer também nos seres humanos ${ }^{2}$.

Os HPAs são formados durante a combustão incompleta ou pirólise de materiais contendo carbono e hidrogênio. Quando emitidos em atmosferas poluídas, especialmente quando ocorre um episódio de smog fotoquímico, os HPAs adsorvidos nas partículas são expostos a uma variedade de poluentes gasosos. Estes incluem intermediários reativos tais como radicais livres e espécies moleculares no estado excitado e estáveis.

Como a queima da biomassa é sempre incompleta, os hidrocarbonetos policíclicos aromáticos são formados como subprodutos.

Em áreas florestais, poucos estudos têm sido realizados com o objetivo de determinar a concentração dos HPAs no material particulado ${ }^{3,4}$.

As emissões das queimadas variam com o tipo de combustível utilizado, intensidade do fogo e fase da combustão (smoldering ou flaming). A fase flaming é caracterizada pela combustão com chamas altas (temperaturas de $340^{\circ} \mathrm{C}$ ou maiores); a fase smoldering, com temperaturas mais baixas, é caracterizada pela combustão incompleta, sem chamas ou com pequenas chamas onde a conversão pirolítica da biomassa, sem oxigênio, predomina ${ }^{5}$.

Os HPAs são emitidos principalmente na forma gasosa, mas uma porção significante, os HPAs mais pesados, estão associados às partículas finas carbonadas.

Os HPAs podem ser convertidos nos seus derivados nitrados quando expostos a agentes nitrantes. Entre eles, os particularmente ativos são $\mathrm{NO}, \mathrm{HNO}_{3}, \mathrm{~N}_{2} \mathrm{O}_{5}$ e radicais $\mathrm{NO}_{3}$. Os dois primeiros são capazes de converter os HPAs adsorvidos nas partículas, enquanto $\mathrm{N}_{2} \mathrm{O}_{5}$ e os radicais $\mathrm{NO}_{3}$ reagem exclusivamente com os HPAs na fase gasosa ${ }^{6}$.

Um mecanismo citado no trabalho de Pitts $^{7}$ é proposto: fluoranteno $(\mathrm{Fa})$ e pireno $(\mathrm{Pi})$ reagem primeiramente com radicais $\mathrm{OH}$ gerando espécies intermediárias hidroxiladas. A adição de $\mathrm{NO}_{2}$ à posição de maior densidade eletrônica (posições 2, 7 e 8 para o fluoranteno; posições 2 e 4 para pireno) seguida pela perda de água conduz aos produtos finais. A mesma reação com $\mathrm{NO}_{3}$, gera radicais nitratociclodienilas seguida pela perda de uma molécula de ácido nítrico. O esquema dos mecanismos é apresentado na figura 1.

Outro estudo feito em laboratório ${ }^{8}$ sobre as reações em fase gasosa do radical $\mathrm{OH}$ com $\mathrm{Fa}$ e $\mathrm{Pi}$ na presença de $\mathrm{NO}_{\mathrm{x}}$ também mostrou que essas reações poderiam ser importantes vias de formação de 2-nitrofluoranteno (2-NFa) e 2-nitropireno (2-NPi) observadas na atmosfera. Os experimentos também foram realizados em câmara de teflon, simulando uma atmosfera real.

A possibilidade do uso dos isômeros 2-NFa e 2-NPi como indicadores de poluição fotoquímica e de emissões antropogênicas está ligada ao fato que alguns deles são diretamente emitidos pelas fontes veiculares, enquanto outros são formados por reações em fase gasosa dos seus HPAs precursores com radicais $\mathrm{OH}$ e $\mathrm{NO}_{3}$ na presença de $\mathrm{NO}_{2}{ }^{9}$. Em áreas urbanas altamente sujeitas à poluição fotoquímica, 2-NFa pode ser responsável por $10 \%$ de toda atividade mutagênica associada ao material particulado. Entretanto, em áreas florestais onde ocorre a queima de biomassa, nenhum estudo ainda foi realizado.

Para verificar a formação dos isômeros 2-NFa e 2-NPi e 


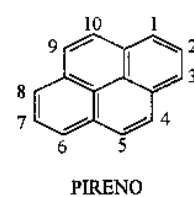<smiles>CO</smiles>

PIRENO<smiles>O=[N+]([O-])c1cc2ccc3cccc4ccc(c1)c2c34</smiles>

$\underline{\mathrm{H}_{2} \mathrm{O}}$

2-NITROPIRENO<smiles>CCC(=O)ON=c1c2cccc3cccc(c4cccc5cccc1c54)c32</smiles><smiles>CO</smiles>

$$
\text { FLUORANTENO }
$$

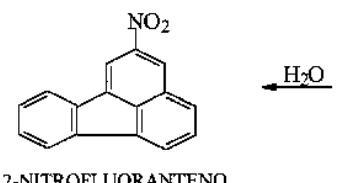

2-NITROFLUORANTENO<smiles>O=[N+]([O-])[O-]</smiles><smiles></smiles>

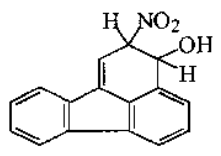

Figura 1. Esquema do mecanismo de formação dos nitro-HPAs segundo James N. Pitts Jr.?.

estabelecer a sua ocorrência na camada limite atmosférica, é importante avaliar a sua presença em áreas remotas onde o material carbonado pode estar presente devido ao transporte das massas de ar e, no caso da Floresta Amazônica, onde a prática da queima de biomassa adotada, gera espécies orgânicas e radicais altamente reativos, propícios para a acentuação da poluição atmosférica regional.

\section{OBJETIVO}

O objetivo deste trabalho é determinar as concentrações dos n-alcanos, HPAs e dos nitro-HPAs 2-nitrofluoranteno e 2nitropireno, visando a avaliação da contribuição antropogênica (as queimadas) e fotoquímica, no estudo dos processos responsáveis pela presença destas espécies na atmosfera de Alta Floresta, Amazônia.

\section{AMOSTRAGEM}

\subsection{Amostragem em Alta Floresta, Amazônia}

\subsubsection{Amostras Ambientais}

Em Alta Floresta, região norte do Estado de Mato Grosso o material particulado total foi coletado em filtros de fibra de quartzo Pallflex $(20 \times 25 \mathrm{~cm})$ usando um amostrador de grande volume (Sierra Andersen, Atlanta, GA, USA). O tempo de amostragem foi de 12 horas, das 7:00 às 19:00 e das 19:00 às 7:00, começando dia 29/08/93 e terminando dia 8/09/93. Todos os filtros foram analisados gravimetricamente antes e depois da amostragem. Após a amostragem, os filtros foram estocados a $-10^{\circ} \mathrm{C}$ para evitar perdas de compostos voláteis.

\subsubsection{Amostras de Emissão Direta}

O material particulado foi coletado utilizando filtros de fibra de quartzo de $47 \mathrm{~mm}$ de diâmetro utilizando um amostrador de pequeno volume. O tempo de amostragem variou de 1 à 3 minutos durante a queima de biomassa nas fases flaming e smoldering e o volume amostrado variou de 16 a 50 litros de ar.

\section{EXTRAÇÃO DO MATERIAL PARTICULADO ATMOSFÉRICO}

Vários métodos de extração do material orgânico particulado do filtro são encontrados na literatura, quase todos utilizando o aparelho de Soxhlet. Já em 1978, Jager ${ }^{10}$ utilizou diclorometano e até hoje tem sido largamente empregado. O método de extração adotado nesse trabalho foi de Ciccioli et al. ${ }^{11}$. O material orgânico particulado foi extraído dos filtros de fibra de quartzo em um aparelho de Soxhlet por 24 horas (60 ciclos) e $150 \mathrm{ml}$ de diclorometano foram usados como solvente para a extração líquida.

Na primeira fase do estudo foi desenvolvida uma metodologia para a separação dos nitro-HPAs, dos hidrocarbonetos alifáticos, HPAs e HPAs oxigenados. Para testar esse novo método, os filtros foram submetidos a uma extração em vez de fazer uma extração em coluna de alumina básica como descrita na literatura ${ }^{12}$. Esse novo método apresenta a vantagem de ser mais simples e rápido.

Após a extração em Soxhlet, o solvente foi evaporado sob fluxo de $\mathrm{N}_{2}$ à temperatura ambiente; depois o extrato foi dissolvido em $1 \mathrm{ml}$ de DMSO e submetido à uma extração líquido-líquido com $1 \mathrm{ml}$ de $\mathrm{n}$-hexano, três vezes, para separar os componentes alifáticos. Então, $3 \mathrm{ml}$ de água foram adicionados e os HPAs, os nitro-HPAs e os oxi-HPAs, foram extraídos numa segunda extração líquido-líquido efetuada com cicloexano (1 $\mathrm{ml}$, tres vezes).

A solução de HPAs e derivados foi separada por cromatografia líquida de alta eficiência (CLAE) numa coluna empacotada com sílica (Erbasil CarloErba, L=250 mm, D=4 mm, $\mathrm{d}=10 \mathrm{~m})$, coletando 3 frações.

A fim de obter condições de retenção reprodutíveis da mistura, a coluna foi lavada com diclorometano (20 min, $1,5 \mathrm{ml} /$ min) e com n-hexano (10 $\mathrm{min}, 1,5 \mathrm{ml} / \mathrm{min}$ ) antes de cada análise. O gradiente de eluição dos compostos tendo diferentes polaridades foi com n-hexano e diclorometano num fluxo de $2 \% \mathrm{v} / \mathrm{v}$ por minuto. O cromatógrafo líquido (Varian modelo 5000) foi conectado a um detector de fluorescência sintonizado na faixa do UV (Varian Associates, Inc., Palo Alto, Ca, USA) em $254 \mathrm{~nm}$.

A fração com os HPAs (Antraceno à Benzo(ghi)perileno) foi obtida entre 30 e $54 \%$ de diclorometano, a fração dos nitroHPAs foi obtida entre 55 e $75 \%$ e a terceira e última fração contendo os oxi-HPAs foi obtida entre 75 e $100 \%$ de diclorometano. Esta metodologia foi testada e adotada devido a maior seletividade das classes químicas, possibilitando melhor separação dos compostos na etapa posterior, com menor número de compostos interferentes ${ }^{13}$. A figura 2 apresenta a metodologia analítica utilizada neste trabalho.

As concentrações das espécies apresentadas nas tabelas são as médias das análises duplicadas e triplicadas. Uma reprodutibilidade típica menor que $0,5 \%$ foi obtida.

\subsection{Materiais}

Diclorometano (DCM), cicloexano, acetona, água, dimetilsulfóxido (DMSO), ortoterfenila, terfenilbenzeno, n-octano, são de grau cromatográfico obtidos da Carlo Erba (Farmitália, Milano, Itália). 1-nitropireno da Aldrich Chemical Co. (Milwaukee, WI) foi purificado de acôrdo com o método de Paputa-Peck et al. ${ }^{14}$; 2-nitropireno foi obtido após repetida purificação de extratos obtidos de aerossóis e vapores emitidos por uma indústria de eletrodos de carbono. 1-, 3-, 7- e 8-nitrofluoranteno foram obtidos pela reação de fluoranteno com ácido nítrico concentrado ${ }^{15}$. Todos os isômeros foram separados por cromatografia líquida em coluna de sílica pelo mesmo procedimento usado para as amostras do particulado descrito abaixo. 


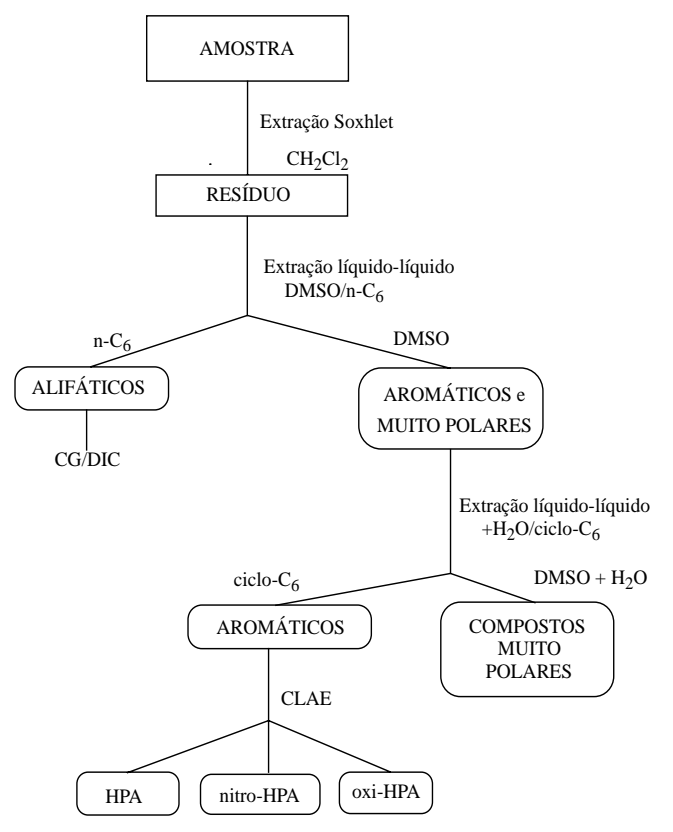

Figura 2. Diagrama de blocos representando a metodologia analítica utilizada ${ }^{11}$.

2-nitrofluoranteno foi obtido pela reação do fluoranteno e $\mathrm{N}_{2} \mathrm{O}_{5}$ em solução de $\mathrm{CCl}_{4}$ à $0{ }^{\circ} \mathrm{C}^{14}$. $\mathrm{O}$ isômero foi identificado pelo espectro de massa e comparado com espectro de RMN descrito na literatura ${ }^{7}$

Os padrões de hidrocarbonetos policíclicos aromáticos foram obtidos da Alfa Products (Denvers, USA), e Jansen Chimica (Beerse, Bélgica).

\section{RESULTADOS E DISCUSSÃO}

\subsection{Amostras Ambientais}

A composição dos n-alcanos e HPAs fornecem informações sobre as características básicas do material particulado presente nos sítios onde os nitro-HPAs são observados, porque estas permitem inferir as principais fontes de emissão, as quais podem ser tanto naturais como antropogênicas. Dados dos nalcanos são também importantes para avaliar se os sítios investigados podem ser considerados representativos das diferentes situações existentes na baixa troposfera.

É citado na literatura ${ }^{16-20}$ que os perfis de distribuição dos alcanos derivados de fontes biológicas (cêras de plantas, solo, etc.), exibem um padrão típico com uma forte predominância das espécies homólogas com número ímpar de carbonos, que pode ser avaliada através do chamado Índice Preferencial de Carbono (I.P.C). O I.P.C. é determinado como sendo a média da soma das concentrações de 2 espécies consecutivas com número ímpar de carbono, sobre o homólogo com número par de carbono. Os alcanos derivados de componentes de combustíveis fósseis (petróleo, carvão, etc.) mostram um perfil sem nenhuma predominância do número de carbonos. A maioria dos produtos oriundos do petróleo contém n-alcanos na faixa de 1 a 35 carbonos, sem predomínio na distribuição ímpar-par dos carbonos, e com uma distribuição assemelhada à gaussiana. Os homólogos $\mathrm{C}_{1}-\mathrm{C}_{20}$ são geralmente perdidos devido à evaporação no ambiente e/ou em procedimentos experimentais (amostragem e manuseio das amostras). Por causa destas características, o perfil dos n-alcanos tem sido utilizado para explicar a origem da componente orgânica dos aerossóis atmosféricos.

A figura 3 apresenta o gráfico das distribuições conforme o número de carbonos das concentrações médias dos alcanos identificados no sítio amostrado.

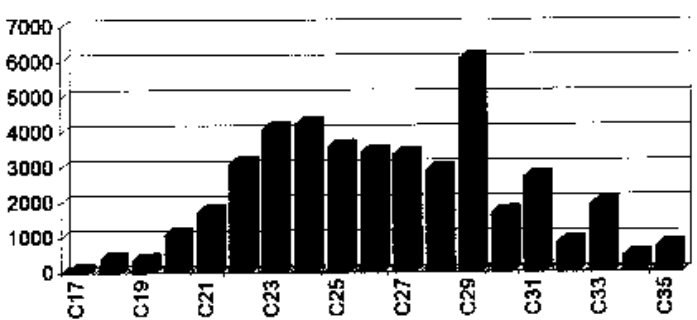

Figura 3. Gráfico das concentrações médias dos n-alcanos identificados nas amostras de Alta Floresta.

As concentrações individuais médias dos alcanos detectados em Alta Floresta variaram de 56 à $6044 \mathrm{pg} / \mathrm{m}^{3}$ (Tabela 1). Os homólogos com mais de 23 carbonos são derivados principalmente de cêras das plantas com predominância dos homólogos com número ímpar de carbonos. Segundo Brodin et al. ${ }^{21}$ tal distribuição também pode ser encontrada quando existe a queima de biomassa.

O Índice Preferencial de Carbono igual a 2,1 na faixa de $\mathrm{C}_{25}-\mathrm{C}_{35}$ indica a origem biológica do material particulado, e o IPC igual a 0,8 na faixa de $\mathrm{C}_{17}-\mathrm{C}_{23}$ indica que houve a influência das emissões das queimadas no material amostrado. Abas et al. $^{4}$ encontraram valores mais altos (3,6 em média) para o I.P.C., calculados na faixa de $\mathrm{C}_{24}-\mathrm{C}_{35}$, de amostras coletadas em Manaus, Amazonas.

Embora apenas 16 amostras ambientais tenham sido coletadas, foi feito um estudo do inter-relacionamento entre os vários compostos orgânicos medidos. Foi utilizada a análise de agrupamento e a análise de fatores principais. $O$ objetivo da análise de agrupamento foi reunir as espécies de acôrdo com as similaridades das variações das concentrações dos HPAs e dos alcanos.

A tabela 2 apresenta os coeficientes de correlação linear normalizados para os n-alcanos. Estes coeficientes foram obtidos utilizando o pacote estatístico Statgraphics versão $6^{21 .}$ As espécies de $C_{23}$ a $C_{30}$ apresentam alta correlação entre si. É proposta a mesma fonte de emissão para todas as espécies em todos os dias amostrados com exceção das amostras AF02. Esta amostra apresentou concentrações discrepantes das demais; o motivo deste comportamento pode ser atribuído às contaminações durante o transporte e/ou devido a umidade verificada no filtro.

As correlações efetuadas entre os HPAs indicaram que os HPAs mais pesados, acima de benzo(ghi)fluoranteno, têm boa correlação entre si (Tabela 3). Os mais leves, Fe+Ant, Fa e Pi, não se correlacionaram bem com os mais pesados. Isto deve ter acontecido devido a distribuição destas espécies entre as fases particulada e gasosa. Distribuição esta, diferente para cada espécie, dependente da temperatura ambiental.

A tabela 4 abaixo apresenta o coeficiente de correlação entre os dois nitroarenos de origem fotoquímica encontrados nestas amostras. A alta correlação confirma os estudos que afirmam que estes compostos são formados pelo mesmo mecanismo de reação e não são emitidos por nenhuma fonte de emissão antropogênica.

Tabela 4. Coeficientes de correlação entre as concentrações do $2-\mathrm{NFa}$ e 2-NPi, das amostras coletadas em Alta Floresta.

\begin{tabular}{lll}
\hline & $2-\mathrm{NPi}$ & $2-\mathrm{NFa}$ \\
\hline $2-\mathrm{NPi}$ & 1 & 0.92 \\
$2-\mathrm{NFa}$ & 0.97 & 1 \\
\hline
\end{tabular}

Embora todos os componentes possam ser explicados pelas emissões das queimadas pode-se supor que a qualquer momento o material particulado possa ter sido trazido por alguma massa 


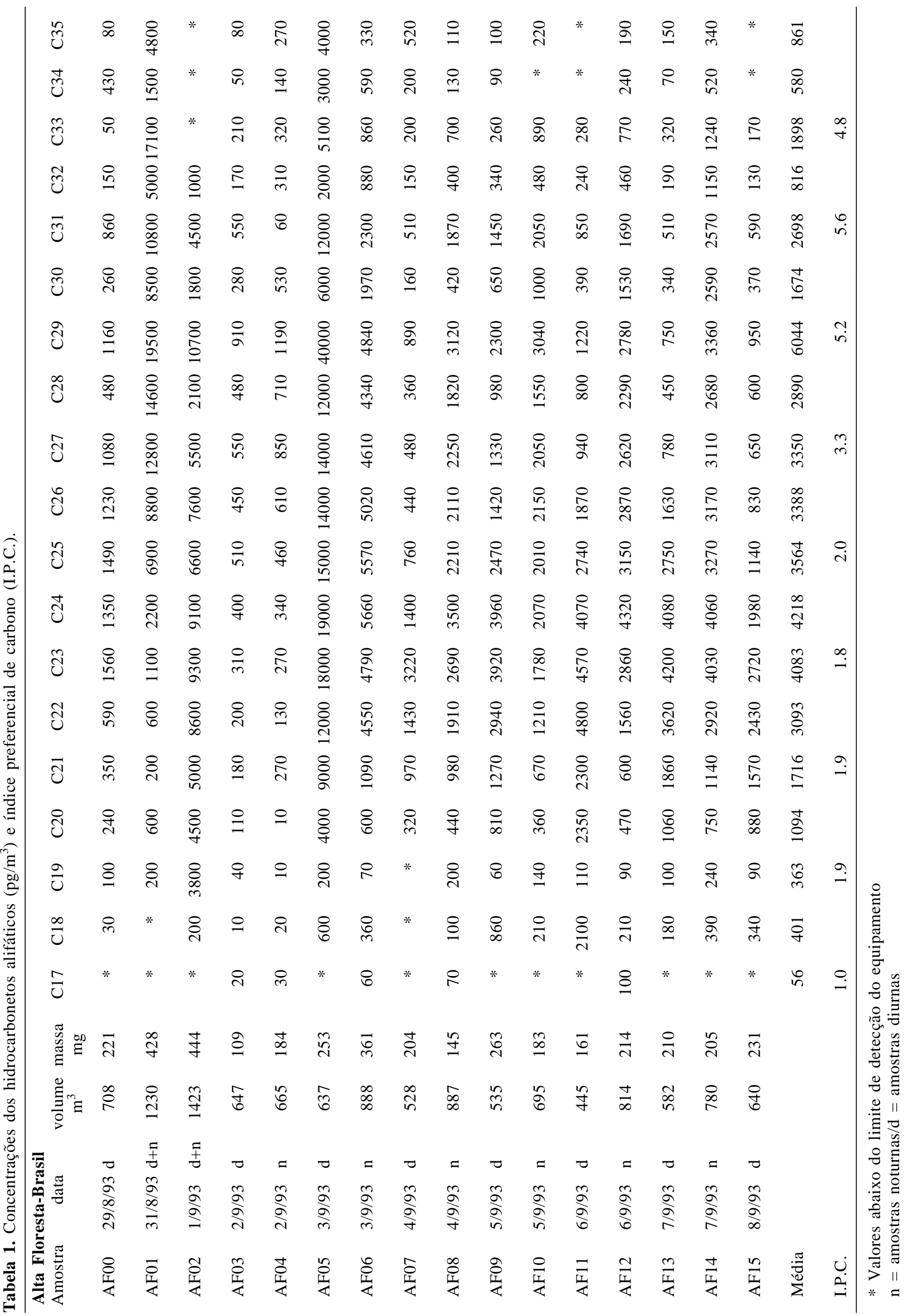


Tabela 2. Coeficientes de correlação entre alguns n-alcanos identificados nas amostras de Alta Floresta.

\begin{tabular}{llllllllllllllll}
\hline & C19 & C20 & C21 & C22 & C23 & C24 & C25 & C26 & C27 & C28 & C29 & C30 & C31 & C32 & C33 \\
\hline C19 & 1,00 & 0,69 & 0,41 & 0,48 & 0,35 & 0,32 & 0,26 & 0,34 & 0,18 & 0,01 & 0,16 & 0,06 & 0,18 & 0,02 & 0,07 \\
C20 & 0,69 & 1,00 & 0,90 & 0,92 & 0,86 & 0,82 & 0,73 & 0,70 & 0,51 & 0,31 & 0,63 & 0,31 & 0,52 & 0,13 & 0,04 \\
C21 & 0,41 & 0,90 & 1,00 & 0,96 & 0,98 & 0,95 & 0,84 & 0,84 & 0,60 & 0,41 & 0,79 & 0,37 & 0,61 & 0,11 & 0,05 \\
C22 & 0,48 & 0,92 & 0,96 & 1,00 & 0,99 & 0,95 & 0,83 & 0,83 & 0,56 & 0,37 & 0,71 & 0,33 & 0,55 & 0,11 & 0,01 \\
C23 & 0,35 & 0,85 & 0,98 & 0,97 & 1,00 & 0,98 & 0,88 & 0,88 & 0,62 & 0,45 & 0,80 & 0,40 & 0,63 & 0,15 & 0,05 \\
C24 & 0,32 & 0,82 & 0,95 & 0,95 & 0,98 & 1,00 & 0,92 & 0,92 & 0,69 & 0,53 & 0,84 & 0,47 & 0,69 & 0,22 & 0,13 \\
C25 & 0,26 & 0,73 & 0,84 & 0,83 & 0,88 & 0,92 & 1,00 & 1,00 & 0,91 & 0,80 & 0,95 & 0,76 & 0,90 & 0,45 & 0,47 \\
C26 & 0,34 & 0,70 & 0,77 & 0,75 & 0,79 & 0,85 & 0,98 & 0,98 & 0,96 & 0,86 & 0,95 & 0,84 & 0,95 & 0,55 & 0,59 \\
C27 & 0,18 & 0,51 & 0,59 & 0,56 & 0,62 & 0,69 & 0,91 & 0,91 & 1,00 & 0,97 & 0,94 & 0,95 & 0,99 & 0,69 & 0,78 \\
C28 & 0,01 & 0,31 & 0,41 & 0,37 & 0,45 & 0,53 & 0,80 & 0,80 & 0,97 & 1,00 & 0,86 & 0,98 & 0,96 & 0,76 & 0,89 \\
C29 & 0,16 & 0,63 & 0,79 & 0,71 & 0,80 & 0,84 & 0,95 & 0,95 & 0,04 & 0,86 & 1,00 & 0,81 & 0,95 & 0,53 & 0,58 \\
C30 & 0,06 & 0,31 & 0,37 & 0,33 & 0,39 & 0,47 & 0,76 & 0,76 & 0,95 & 0,98 & 0,81 & 1,00 & 0,94 & 0,79 & 0,91 \\
C31 & 0,18 & 0,52 & 0,61 & 0,55 & 0,63 & 0,69 & 0,90 & 0,90 & 0,99 & 0,96 & 0,95 & 0,94 & 1,00 & 0,71 & 0,79 \\
C32 & 0,02 & 0,13 & 0,11 & 0,11 & 0,15 & 0,22 & 0,48 & 0,55 & 0,69 & 0,76 & 0,53 & 0,79 & 0,71 & 1,00 & 0,81 \\
C33 & 0,07 & 0,04 & 0,05 & 0,01 & 0,05 & 0,13 & 0,47 & 0,59 & 0,78 & 0,89 & 0,58 & 0,91 & 0,79 & 0,79 & 1,00 \\
\hline
\end{tabular}

Tabela 3. Coeficiente de correlação entre alguns HPAs identificados nas amostras de Alta Floresta.

\begin{tabular}{lccccccccccccc}
\hline & $\mathrm{Fe}+$ Ant & $\mathrm{Fa}$ & $\mathrm{Pi}$ & $\mathrm{BghiFa}$ & $\mathrm{BaAnt}$ & $\mathrm{Cri}$ & $\mathrm{BbjkFa}$ & $\mathrm{BaFa}$ & $\mathrm{BePi}$ & $\mathrm{BaPi}$ & InPi & DBaAnt BghiPer \\
\hline Fe+Ant & 1,00 & 0,45 & 0,68 & 0,18 & 0,01 & 0,01 & 0,01 & 0,18 & 0,01 & 0,01 & 0,01 & 0,01 & 0,01 \\
$\mathrm{Fa}$ & 0,46 & 1,00 & 0,56 & 0,19 & 0,07 & 0,10 & 0,13 & 0,10 & 0,08 & 0,01 & 0,09 & 0,03 & 0,09 \\
$\mathrm{Pi}$ & 0,68 & 0,56 & 1,00 & 0,75 & 0,35 & 0,43 & 0,31 & 0,12 & 0,27 & 0,21 & 0,20 & 0,34 & 0,31 \\
$\mathrm{BghiFa}$ & 0,18 & 0,19 & 0,75 & 1,00 & 0,56 & 0,68 & 0,50 & 0,01 & 0,48 & 0,4 & 0,42 & 0,51 & 0,65 \\
BaAnt & 0,01 & 0,07 & 0,35 & 0,56 & 1,00 & 0,98 & 0,90 & 0,81 & 0,88 & 0,86 & 0,86 & 0,76 & 0,89 \\
Cri & 0,01 & 0,10 & 0,43 & 0,68 & 0,98 & 1,00 & 0,94 & 0,70 & 0,91 & 0,89 & 0,88 & 0,81 & 0,94 \\
BbjkFa & 0,03 & 0,13 & 0,31 & 0,50 & 0,90 & 0,94 & 1,00 & 0,69 & 0,99 & 0,97 & 0,95 & 0,89 & 0,92 \\
BaFa & 0,01 & 0,01 & 0,01 & 0,01 & 0,81 & 0,70 & 0,69 & 1,00 & 0,67 & 0,68 & 0,68 & 0,54 & 0,60 \\
BePi & 0,04 & 0,08 & 0,27 & 0,48 & 0,88 & 0,91 & 0,99 & 0,67 & 1,00 & 0,99 & 0,96 & 0,88 & 0,93 \\
BaPi & 0,01 & 0,01 & 0,21 & 0,44 & 0,86 & 0,89 & 0,97 & 0,68 & 0,99 & 1,00 & 0,96 & 0,90 & 0,92 \\
InPi & 0,17 & 0,09 & 0,20 & 0,42 & 0,86 & 0,88 & 0,95 & 0,68 & 0,96 & 0,96 & 1,00 & 0,90 & 0,94 \\
DBAnt & 0,08 & 0,03 & 0,34 & 0,51 & 0,76 & 0,81 & 0,89 & 0,54 & 0,88 & 0,90 & 0,90 & 1,00 & 0,83 \\
BghiPer & 0,18 & 0,09 & 0,31 & 0,65 & 0,89 & 0,94 & 0,92 & 0,60 & 0,93 & 0,92 & 0,94 & 0,83 & 1,00 \\
\hline
\end{tabular}

Tabela 5. Concentrações dos hidrocarbonetos policíclicos aromáticos $\left(\mathrm{pg} / \mathrm{m}^{3}\right)$.

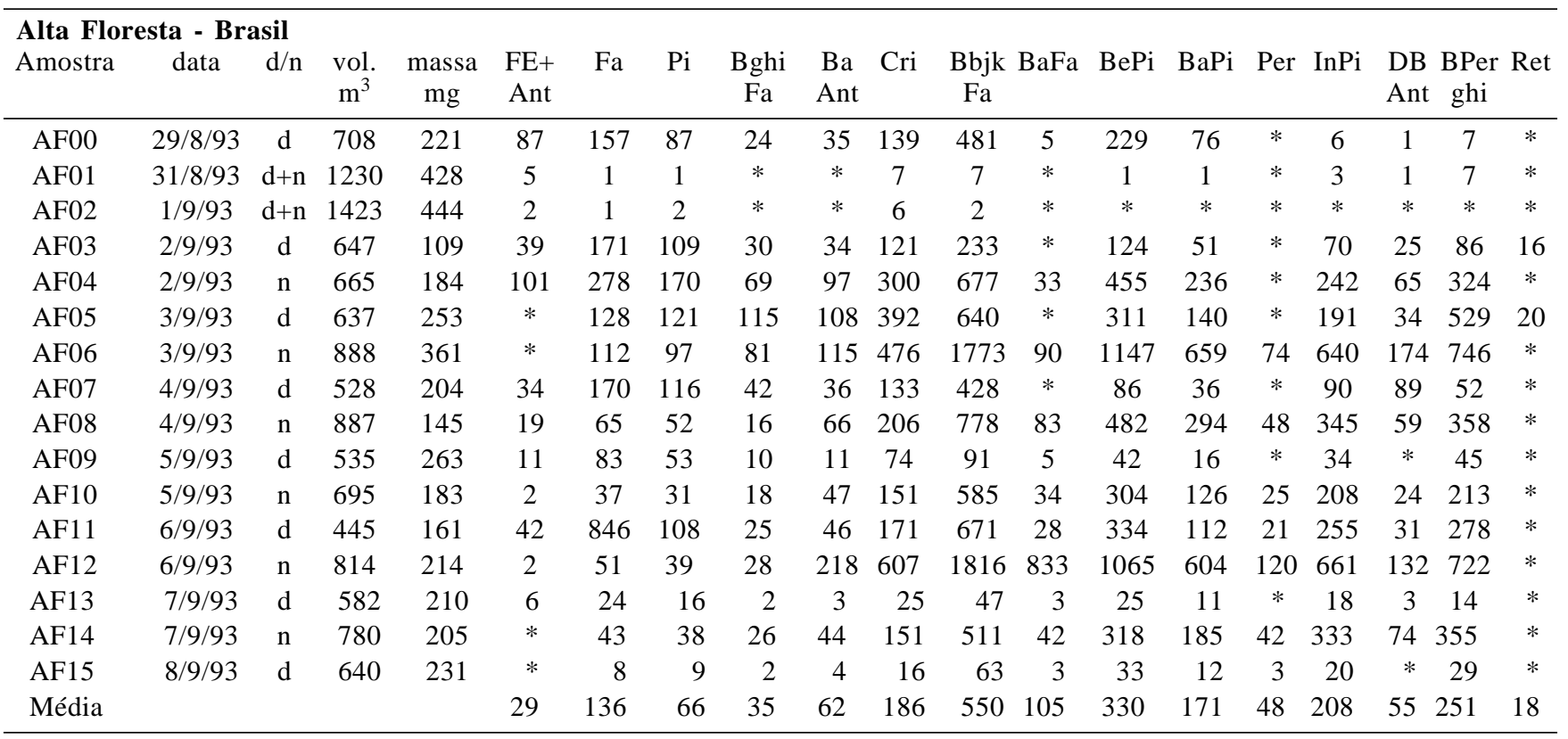


de ar e que devido às reatividades das diferentes classes de compostos, as reações fotoquímicas com oxigênio e com poluentes secundários produzidos pela fotólise, tais como ozônio, PAN e radicais hidroxilas e hidroperoxilas, tenham influenciado a composição do material particulado. Também é válido considerar que devido as queimadas, a concentração das espécies não medidas como $\mathrm{NO}_{x}, \mathrm{CO}, \mathrm{CH}_{4}$, entre outras, podem ter influenciado na razão de formação de alguns dos compostos estudados.

Aplicando nas amostras a análise de agrupamentos utilizando o software SPSS (Statistical Package for Social Sciences), os resultados revelam alguns agrupamentos significantes (Figura 4). Os alcanos $C_{20}$ ao $C_{32}$ se mostraram altamente relacionados entre si. Os resultados das análises de fatores é em geral compatível com o resultado da análise de grupamentos. Pela análise de agrupamentos foram observados 3 grupos distintos de compostos: o primeiro correspondendo aos n-alcanos $\mathrm{C}_{20}-\mathrm{C}_{32}$; o segundo agrupando pireno, benzo(ghi)fluoranteno, fluoranteno e a concentração mássica do material coletado; o terceiro grupo compõe-se do benzo(a)antraceno, criseno, benzo(e)pireno, benzo(a)pireno, benzo(bjk)fluoranteno, indeno(cd)pireno e benzo(ghi)perileno.

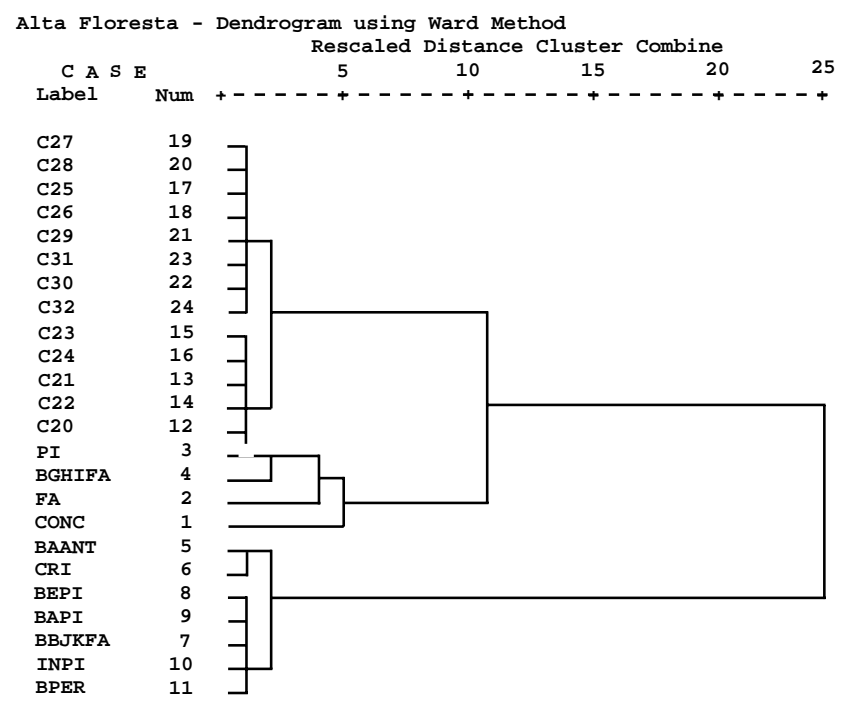

Figura 4. Dendograma apresentando os agrupamentos obtidos utilizando o software SPSS.

As concentrações médias das espécies individuais dos HPAs detectados nas amostras de Alta Floresta variaram de 29 a 550 $\mathrm{pg} / \mathrm{m}^{3}$ (Tabela 5 e Figura 5).

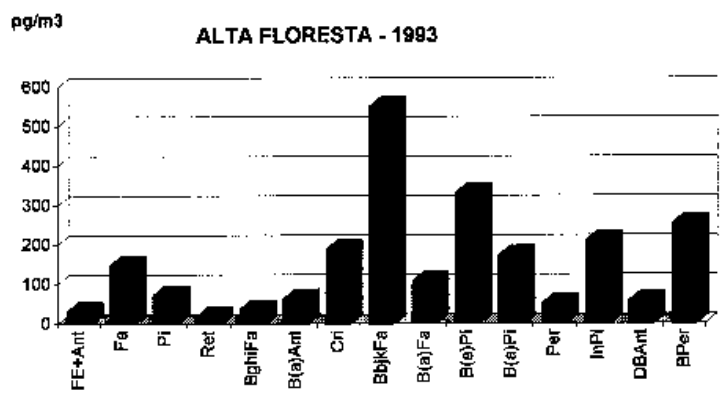

Figura 5. Gráfico das concentrações médias dos HPAs identificados nas amostras de Alta Floresta.

Entre eles estão presentes alguns considerados carcinogênicos: fluoranteno, benzo(a)antraceno, benzo(e)pireno, indeno(cd)pireno, dibenzo(ah)antraceno e, benzo(a)pireno que é considerado mutagênico.
Os seis mais abundantes HPAs detectados nessas amostras foram: benzo(bjk)fluoranteno, benzo(e)pireno, benzo(ghi)perileno, indeno(cd)pireno, criseno e benzo(a)pireno. Alguns destes compostos não podem ser considerados traçadores específicos de produtos da queima de biomassa desde que eles são encontrados em todos os tipos de combustão (industrial, usinas geradoras de energia, queima de petróleo ou diesel). Isto se aplica por exemplo ao Fa que é abundante em todas as fontes de emissões antropogênicas. Em queimadas, as concentrações de criseno e pireno são muito abundantes ${ }^{3}$.

$\mathrm{O}$ aging das partículas na atmosfera pode ser estimado ao se calcular a razão benzo(a)antraceno/criseno. Estes compostos de mesmo peso molecular e volatilidade, têm diferentes tempos de permanência na atmosfera. Essa relação pode indicar se o material foi emitido num período menor ou maior do que 3 dias ${ }^{11}$. Observando estas razões calculadas para as amostras de Alta Floresta (Tabela 6) pode-se estimar que o material coletado foi emitido na época de amostragem por não ter havido um decréscimo contínuo nas razões. Decréscimos nessas relações devem ocorrer sempre que partículas estejam expostas à luz do sol e aos agentes químicos por um período maior que 3 dias.

Uma relação importante é benzo(e)pireno/benzo(a)pireno quando se comparam amostras florestais com amostras urbanas, onde essa razão é próxima da unidade nas partículas provenientes de emissões de motores a gasolina, diesel e para queima óleo $^{2}$. No material particulado coletado na floresta, estas razões nas amostras diurnas foram de 2,2 a 3,0 e nas amostras 1,6 a 2,4 (Tabela 6). Pode-se supor que estas razões altas sejam devidas às queimadas. Masclet et al. ${ }^{3}$ sugerem que devido à alta reatividade do benzo(a)pireno e à baixa reatividade do benzo(e)pireno esta razão relaciona-se preferencialmente com a distância entre a fonte de emissão e o sítio receptor, do que, a verdadeira natureza da fonte de combustão.

O gráfico das concentrações médias dos nitro-HPAs encontrados nas amostras de Alta Floresta estão apresentadas na figura 6. As concentrações variam de $4 \mathrm{pg} / \mathrm{m}^{3}$ (1-nitropireno) a 17 $\mathrm{pg} / \mathrm{m}^{3}$ (2-nitrofluoranteno) e estão apresentadas na tabela 7.

Na maioria destas amostras está presente 1-nitropireno, o que indica a contribuição dos veículos que utilizam óleo diesel como combustível, ou a formação de artefato no momento da amostragem. Mas esta contribuição é pequena em relação a contribuição do fenômeno fotoquímico indicado pela presença de 2-nitrofluoranteno e 2-nitropireno, compostos conhecidos como mutagênicos e carcinogênicos. As concentrações destes nitro-HPAs nas amostras noturnas são maiores que das amostras diurnas. Dois processos podem ser discutidos e devem ser mais estudados. O primeiro é que devido a diminuição da temperatura e redução da camada de dispersão dos poluentes, maior condensação dos gases ocorre nas partículas. Medidas da temperatura em diferentes alturas tem mostrado claramente que, em muitos casos, a altura equivalente da camada de dispersão dos poluentes durante o dia é mais de 10 vezes maior do que durante a noite.

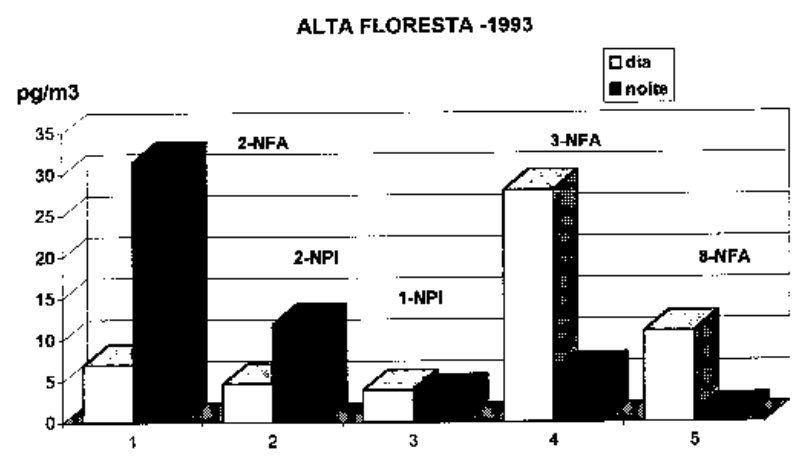

Figura 6. Gráfico das concentrações médias dos nitro-HPAs identificados nas amostras de Alta Floresta. 
Tabela 6. Relações entre as concentrações dos hidrocarbonetos policíclicos aromáticos.

\begin{tabular}{|c|c|c|c|c|c|c|c|c|}
\hline \multicolumn{9}{|c|}{ Alta Floresta - Brasil } \\
\hline Amostra & data & $\mathrm{d} / \mathrm{n}$ & $\mathrm{BePi}$ & $\mathrm{BaPi}$ & $\mathrm{BePi} / \mathrm{BaPi}$ & BaAnt & Cri & $\mathrm{BaAnt} / \mathrm{Cri}$ \\
\hline AF00 & $29 / 8 / 93$ & $\mathrm{~d}$ & 229 & 76 & 3.01 & 35 & 139 & 0.25 \\
\hline AF01 & $31 / 8 / 93$ & $d+n$ & 1 & 1 & 0.92 & $*$ & 7 & $*$ \\
\hline $\mathrm{AF} 02$ & $1 / 9 / 93$ & $\mathrm{~d}+\mathrm{n}$ & $*$ & $*$ & $*$ & $*$ & 6 & $*$ \\
\hline AF03 & 2/9/93 & $\mathrm{d}$ & 124 & 51 & 2.43 & 34 & 121 & 0.28 \\
\hline AF04 & $2 / 9 / 93$ & $\mathrm{n}$ & 455 & 236 & 1.93 & 97 & 300 & 0.32 \\
\hline AF05 & $3 / 9 / 93$ & $\mathrm{~d}$ & 311 & 140 & 2.22 & 108 & 392 & 0.28 \\
\hline AF06 & $3 / 9 / 93$ & $\mathrm{n}$ & 1147 & 659 & 1.74 & 115 & 476 & 0.24 \\
\hline AF07 & $4 / 9 / 93$ & $\mathrm{~d}$ & 86 & 36 & 2.39 & 36 & 133 & 0.27 \\
\hline AF08 & $4 / 9 / 93$ & $\mathrm{n}$ & 482 & 294 & 1.64 & 66 & 206 & 0.32 \\
\hline AF09 & $5 / 9 / 93$ & $\mathrm{~d}$ & 42 & 16 & 2.63 & 11 & 74 & 0.15 \\
\hline AF10 & $5 / 9 / 93$ & $\mathrm{n}$ & 304 & 126 & 2.41 & 47 & 151 & 0.31 \\
\hline AF11 & $6 / 9 / 93$ & $\mathrm{~d}$ & 334 & 112 & 2.98 & 46 & 171 & 0.27 \\
\hline AF12 & 6/9/93 & $\mathrm{n}$ & 1065 & 604 & 1.76 & 218 & 607 & 0.36 \\
\hline AF13 & 7/9/93 & $\mathrm{d}$ & 25 & 11 & 2.27 & 3 & 25 & 0.12 \\
\hline AF14 & $7 / 9 / 93$ & $\mathrm{n}$ & 318 & 185 & 1.72 & 44 & 151 & 0.29 \\
\hline AF15 & $8 / 9 / 93$ & $\mathrm{~d}$ & 33 & 12 & 2.75 & 4 & 16 & 0.25 \\
\hline Média & & & & & 2.19 & & & \\
\hline
\end{tabular}

Tabela 7. Concentrações dos hidrocarbonetos policíclicos aromáticos nitrados $\left(\mathrm{pg} / \mathrm{m}^{3}\right)$.

\begin{tabular}{|c|c|c|c|c|c|c|c|c|c|c|}
\hline $\begin{array}{l}\text { Alta Flor } \\
\text { Amostra }\end{array}$ & $\begin{array}{c}\text { a - Brasil } \\
\text { data }\end{array}$ & dia/noite & $\underset{\mathrm{m}^{3}}{\text { volume }}$ & $\begin{array}{c}\text { massa } \\
\mathrm{mg}\end{array}$ & $2-\mathrm{NFa}$ & 1-NPi & 2-NPi & $2 \mathrm{NFa} / 2 \mathrm{NPi}$ & 3-NFA & 8-NFA \\
\hline AF00 & $29 / 8 / 93$ & $\mathrm{~d}$ & 708 & 221 & 10 & 16 & 2 & $*$ & $*$ & \\
\hline AF01 & $31 / 8 / 93$ & $d+n$ & 1230 & 428 & 1 & $*$ & 1 & 1 & $*$ & $*$ \\
\hline AF02 & $1 / 9 / 93$ & $d+n$ & 1423 & 444 & 9 & 2 & 11 & 1 & $*$ & $*$ \\
\hline AF03 & 2/9/93 & d & 647 & 109 & 8 & 7 & 6 & 1 & $*$ & $*$ \\
\hline AF04 & 2/9/93 & $\mathrm{n}$ & 665 & 184 & 14 & 3 & 11 & 1 & 11 & $*$ \\
\hline AF05 & 3/9/93 & $d$ & 637 & 253 & 6 & 4 & 7 & 1 & 28 & 11 \\
\hline AF06 & 3/9/93 & $\mathrm{n}$ & 888 & 361 & 9 & 1 & 1 & 9 & 1 & 1 \\
\hline AF07 & 4/9/93 & d & 528 & 204 & 4 & 1 & 1 & 4 & $*$ & $*$ \\
\hline AF08 & 4/9/93 & $\mathrm{n}$ & 887 & 145 & 25 & $*$ & 10 & 3 & $*$ & $*$ \\
\hline AF09 & $5 / 9 / 93$ & $\mathrm{~d}$ & 535 & 263 & $*$ & $*$ & $*$ & & $*$ & $*$ \\
\hline AF10 & $5 / 9 / 93$ & $\mathrm{n}$ & 695 & 183 & 24 & 6 & 10 & 2 & $*$ & $*$ \\
\hline AF11 & 6/9/93 & d & 445 & 161 & 9 & 7 & 7 & 1 & $*$ & $*$ \\
\hline AF12 & 6/9/93 & $\mathrm{n}$ & 814 & 214 & 97 & $*$ & 29 & 3 & $*$ & $*$ \\
\hline AF13 & 7/9/93 & d & 582 & 210 & 5 & $*$ & 1 & 5 & $*$ & $*$ \\
\hline AF14 & 7/9/93 & $\mathrm{n}$ & 780 & 205 & 20 & $*$ & 10 & 2 & $*$ & $*$ \\
\hline AF15 & $8 / 9 / 93$ & $\mathrm{~d}$ & 640 & 231 & $*$ & $*$ & $*$ & & $*$ & $*$ \\
\hline Média & & & & & 17 & 4 & 8 & & 13 & 6 \\
\hline
\end{tabular}

* Valores abaixo do limite de detecção do equipamento

$\mathrm{n}=$ amostras noturnas $/ \mathrm{d}=$ amostras diurnas

Outro processo que merece ser discutido é a fotodecomposição que os nitroarenos sofrem durante sua exposição à luz solar. Estudos recentes ${ }^{22}$ mostram que esta fotodecomposição parece estar fortemente relacionada à natureza química e física dos substratos aos quais os nitroarenos estão ligados.

É interessante observar as relações 2-nitrofluoranteno/2nitropireno encontradas nas amostras (Tabela 7). O valor $(2,6$ em média) é muito menor do que os encontrados normalmente em qualquer sítio urbano já estudado (sempre acima de 4). $\mathrm{O}$ que se pode sugerir é que com a grande emissão de pireno e ozônio pelas queimadas, uma grande produção de radicais hidroxila é verificada pela fotólise do ozônio, favorecendo maior formação de 2-nitropireno.

As espécies 3- e 8-nitrofluoranteno foram encontradas nas amostras de Alta Floresta. Estes compostos são raramente encontrados em áreas remotas, não são compostos de origem fotoquímica e podem ter sido formados no momento da amostragem (artefato).

A terceira fração separada pela CLAE, contém os oxi-HPAs oxigenados, que foram parcialmente identificados por CG/DIC. Em todas as amostras coletadas foi observada a presença de ftalatos, entre muitos outros compostos. Estas espécies foram identificadas ao se comparar os tempos de retenção das espécies das amostras com os tempos da solução padrão de ftalatos. Sua presença confirma a idéia de que esses compostos são transportados e podem ser encontrados em zonas remotas ${ }^{23}$. Mais estudos no entanto, devem ser feitos para esclarecer a sua presença em áreas florestais e remotas. 


\subsection{Amostras coletadas diretamente sôbre as queimadas (flaming e smoldering)}

O perfil das concentrações dos n-alcanos das amostras coletadas nas fases smoldering e flaming é apresentado nos gráficos das figuras 7 e 8 respectivamente. As concentrações de 8 amostras da fase smoldering extraídas simultaneamente variaram de $58 \mathrm{ng} / \mathrm{m}^{3}$ a $1550 \mathrm{ng} / \mathrm{m}^{3}$, e de 2 amostras da fase flaming variaram de 379 a $20003 \mathrm{ng} / \mathrm{m}^{3}$. Nestas amostras não foi observada nenhuma predominância dos números ímpares ou pares dos carbonos nas distribuições dos homólogos.

Quanto aos HPAs, o criseno foi particularmente abundante em fumaças de queima de biomassa nas fases smoldering e flaming (Figuras 9 e 10) sendo considerado um dos traçadores dos produtos desses tipo de queima em amostras coletadas em cerrados $^{3}$. Os nitroarenos 2-nitrofluoranteno e 2-nitropireno não foram identificados, consolidando a teoria que eles são formados por reações fotoquímicas e não são emitidos diretamente.

Os três perfis apresentados nas figuras 5, 9 e 10 (amostras ambientais, flaming e smoldering), diferem pouco qualitativamente. Em apenas duas das amostras ambientais foi determinado o reteno. Este composto é um produto característico da combustão de madeira, sendo formado pela degradação térmica de compostos da resina das plantas especialmentede coníferas ${ }^{24}$.

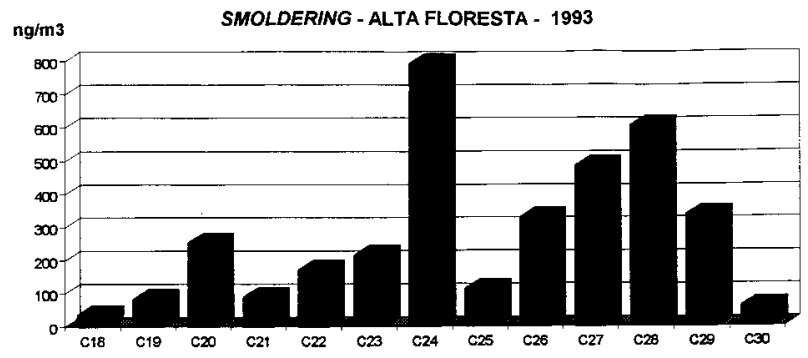

Figura 7. Gráfico das concentrações médias dos alcanos identificados nas amostras coletadas na fase smoldering.

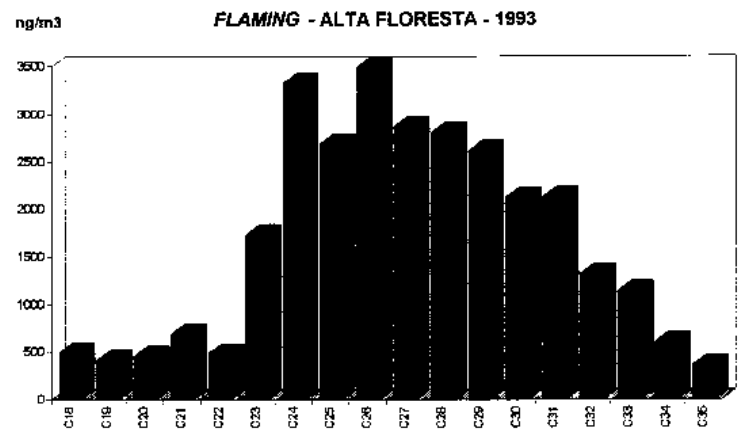

Figura 8. Gráfico das concentrações médias dos n-alcanos identificados nas amostras coletadas na fase flaming.

\section{CONCLUSÕES}

A grande variação da concentração dos nitroarenos 2 nitrofluoranteno e 2-nitropireno que foram observadas nesta área florestal, refletiu bem o equilíbrio complexo entre os processos de formação e de remoção aos quais os nitro-HPAs foram submetidos na atmosfera. Enquanto a formação dos nitros-HPAs depende dos níveis dos HPAs gasosos e $\mathrm{NO}_{2}$ às quais são expostas durante o tempo em que estão suspensas no ar, sua remoção é causada pela deposição das partículas na superfície da terra e, degradação fotolítica dos compostos. Desde que a fotólise e a deposição afetam todos os nitroarenos presentes na partícula numa mesma extensão, diferenças nas relações

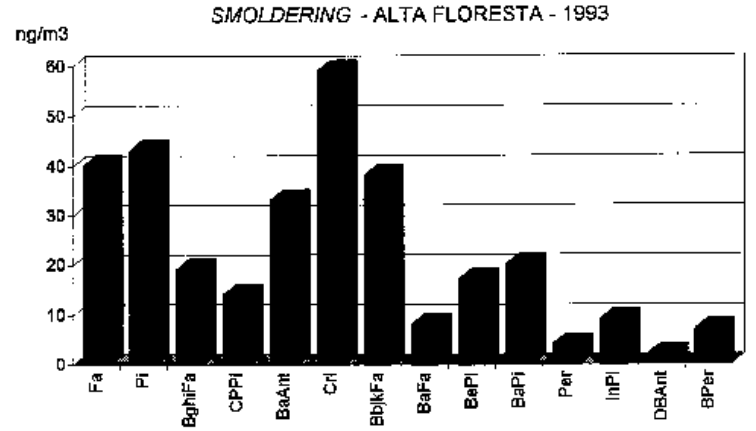

Figura 9. Gráfico das concentrações médias dos HPAs identificados nas amostras coletadas na fase smoldering.

FLAMNG - ALTA FLORESTA - 1993

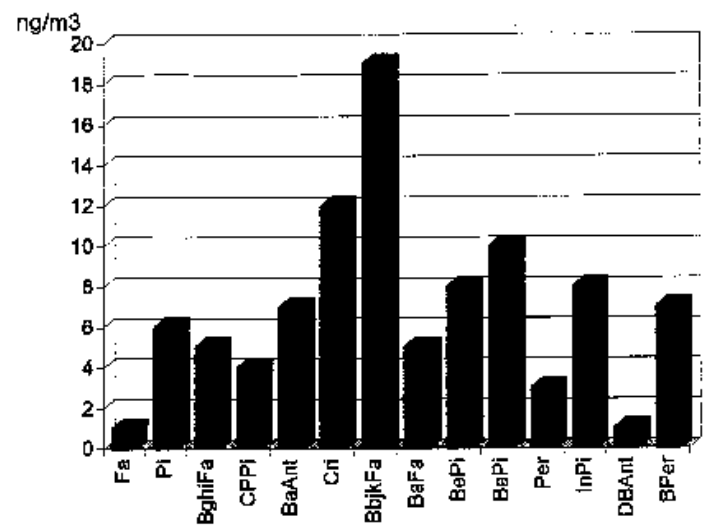

Figura 10. Gráfico das concentrações médias dos HPAs identificados nas amostras coletadas na fase flaming.

2-nitrofluoranteno/2-nitropireno não deveriam ser observadas longe das fontes de emissão.

Quando poluentes gerados em áreas urbanas estão dispersos no ar onde existem baixas emissões de NO, uma grande formação de ozônio ocorre. Sua fotólise se torna uma grande fonte diurna de radicais $\mathrm{OH}$. Esta produção é tão eficaz que a formação dos nitroarenos 2-nitrofluoranteno e 2-nitropireno pode ocorrer mesmo em áreas florestais onde é baixa a emissão de poluentes primários. Entretanto, existe pouco conhecimento sôbre essa formação em áreas florestais.

$\mathrm{Na}$ média, concentrações baixas de 2-nitrofluoranteno foram encontradas nas amostras de Alta Floresta, com razões 2nitrofluoranteno/2-nitropireno por volta de 7. Como pode ser observado, não houve um acréscimo acentuado nas razões benzo(e)pireno/benzo(a)pireno, do que se conclui que as partículas não são envelhecidas; isto indica que os nitroarenos foram depositados em partículas "frescas". Isto sugere que a formação ocorreu não muito longe (20-30 Km) do sítio de amostragem e, provavelmente, quando a queima da floresta estimulou a formação de ozônio.

Como as diferenças nas emissões e processos de remoção não justificam as grandes variações nas relações 2-nitrofluoranteno/2-nitropireno, uma fonte adicional de radicais $\mathrm{OH}$ pode ser estimada. Desde que a fotólise do $\mathrm{HNO}_{2}$ durante o dia pode gerar radicais $\mathrm{OH}$ que se acumulam à noite, tentativas foram feitas para correlacionar a formação e os níveis noturnos dos nitroarenos. Estudos de Ciccioli et al. ${ }^{25}$ correlacionam as concentrações de $\mathrm{HNO}_{2}$ em função das concentrações de 2-nitrofluoranteno encontradas nos dias seguintes daquelas amostragens. A alta similaridade das tendências levam a crer que a 
fotólise do $\mathrm{HNO}_{2}$ pode contribuir para a formação dos nitroarenos, quando concentrações noturnas de $\mathrm{HNO}_{2}$ forem maiores que $10 \mathrm{ug} / \mathrm{m}^{3}$. Esses resultados são sustentados com observações que mostram níveis máximos de 2-nitrofluoranteno encontrados em Milão quando concentrações altas noturnas de $\mathrm{HNO}_{2}$ foram encontradas.

Altas emissões de poluentes primários e condições estáveis numa inversão noturna, explicariam a alta formação de $\mathrm{HNO}_{2}$. Em atmosfera úmida rica em $\mathrm{NO}_{2}$ e pobre em $\mathrm{O}_{3}$, ocorre a oxidação de $\mathrm{NO}_{2}$ à $\mathrm{NO}_{3}$ e a reação heterogênea deste composto com água forma $\mathrm{HNO}_{2}$ e $\mathrm{HNO}_{3}$.

Se existe uma concentração noturna alta de $\mathrm{NO}_{2}, o \mathrm{HNO}_{2}$ pode ser produzido se a razão área/volume for favorável à reação, desde que, a produção do $\mathrm{HNO}_{2}$ é linearmente dependente da superfície disponível à reação heterogênea e à concentração de $\mathrm{NO}_{2}{ }^{26}$.

Outra evidência do papel exercido pelo material particulado na produção de $\mathrm{HNO}_{2}$ e $\mathrm{HNO}_{3}$ é demonstrado pela presença de nitro-HPAs formados pela reação heterogênea com $\mathrm{HNO}_{3}$ no material particulado total coletado na Amazônia. 3- e 8-nitrofluoranteno foram encontrados nas partículas grandes e não nas emissões diretas (flaming e smoldering), sugerindo assim a sua formação por reação heterogênea nas partículas durante a coleta (artefato de amostragem).

\section{REFERÊNCIAS}

1. Artaxo, P.; Maenhaut, W.; J. Geophys. Res. 1988, 93, 1605.

2. Grimmer, G. In: Environmental Carcinogens Polycyclic Aromatic Hydrocarbons. Chemistry, Biochemistry, Carcinogenicity. Edited by Gernot Grimmer 1983.

3. Masclet, P.; Cachier, H.; Liousse, C.; Wortham, H.; J. Atmos. Chem. 1995, 22, 41.

4. Abas, M. R. B.; Simoneit, B. R. T.; Elias, V.; Cabral, J. A.; Cardoso, J. N.-; Chemosphere 1987, 30, 995.

5. Lobert, J. M.; Scharffe, D. H.; Hao, W. M.; Kuhlbusch, T. A.; Seuwen, R.; Warneck, P.; Crutzen, P. J. - "Experimental Evaluation of biomass Burning Emissions: Nitrogen and Carbon Containing Compounds". In: Global Biomass burning - Atmospheric, Climatic, and Biopheric Implications. Edited by Joel S. Levine. Massachusetts Institute of Technology (1991).

6. Pitts, J. N.; Atmos. Environ. 1987, 21, 2531.

7. Pitts, J. N.; Sweetman, J. A.; Zielinska, B.; Winer, A.; Atkinson, R.; Atmos. Environ. 1985, 19, 1608.
8. Arey, J.; Zielinska, B.; Atkinson, R.; Winer, A. M.; Atmos. Environ. 1987, 21, 1437.

9. Finlayson-Pitts, B. J. and Pitts Jr., J. N. - "Chemistry and mutagenic activity of airborne polycyclic aromatic Hydrocarbons and their derivatives". In: Atmospheric Chemistry: Fundamentals and Experimental Techniques. John Wiley \& Sons. 1986.

10. Jager, J.; J. Chromatogr. 1978, 152, 575.

11. Ciccioli, P.; Cecinato, A.; Brancaleoni, E.; Frattoni, M.; Zacchei, P.; Miguel, A. H.; Vasconcellos, P. C.; J. Geophys. Res. 1996, 101, 19567.

12. Ciccioli, P.; Brancaleoni, E.; Cecinato, A.; Di Palo, C.; Buttini, P.; Liberti, A.; J. Chromatogr. 1986, 351, 451.

13. Vasconcellos, P. C.; Tese de Doutoramento. Instituto de Química, Universidade de São Paulo, 1996.

14. Paputa-Peck, M. C.; Marano, R.; Schetzle, D.; Riley, T. L.; Hampton, C. V.; Prater, T. J.; Skewes, L. M.; Jensen, T. E.; Ruehele, P. H.; Bosck, L. C.; Duncan, W. P.; Anal. Chem. 1983, 55, 1946.

15. Kloetzel, M. C.; King, W.; Menkes, J. M.; J. Am. Chem. Soc. 1955, 78, 1165 .

16. Kadowaki, S.; Environ. Sci. Technol. 1994, 28,129.

17. Simoneit, B. R. T.; Mazurek, M. A.; Aer. Sci. \& Technol. 1989, 10, 267.

18. Simoneit, B. R. T.; Atmos. Environ. 1984, 18, 51.

19. Hildemann, L. M.; Mazurek, M.; Cass, G.R.; Simoneit, B. R.; Aer. Sci. \& Technol. 1994, 20, 303.

20. Broddin, G.; Cautreels,W.; Cauwenberghe, K. V.; Atmos. Environ. 1980, 14, 895.

21. Johnson, R. A.; Winchen, D. W. - In: Applied Multivariate Statistical Analysis. New Jersey. Prentice Hall (1982).

22. Fan, Z.; Kamens, R.; Hu, J.; Zhang, J.; McDow, S.; Environ. Sci. Technol. 1996, 30, 1358.

23. Ciccioli, P.; Cecinato, A,; Brancaleoni, E.; Montagnoli, M.; Allegrini, I.; Intern. J. Environ. Anal. Chem. 1994, 55,47

24. Ramdhal, T.; Nature 1983, 306, 580.

25. Ciccioli, P.; Cecinato, A.; Brancaleoni, E.; Atmos. Environm. 1993, 27, 1261.

26. Lammel, G.; Perner, D.; Warneck, P.; "Nitrous Acid in Mainz: Observation and implication for its formation mechanism". In: Physico Chemical Behaviour of Atmospheric Pollutants, edited by G.Restelli and G. Angeletti, pp. 469-476, Kluwer Academic, Norwell, Mass., 1990. 